OPEN ACCESS

Edited by:

Tzu Pin Lu,

National Taiwan University, Taiwan

Reviewed by:

Crescenzio Francesco Minervini, University of Bari Aldo Moro, Italy

Shaolei Teng,

Howard University, United States

*Correspondence:

Myungshin Kim microkim@catholic.ac.kr

Yonggoo Kim

yonggoo@catholic.ac.kr

${ }^{+}$These authors have contributed equally to this work and

share last authorship

Specialty section: This article was submitted to

Cancer Genetics,

a section of the journal

Frontiers in Oncology

Received: 01 June 2021

Accepted: 21 July 2021

Published: 23 August 2021

Citation:

Lee J, Cho S, Hong S-E, Kang D, Choi H, Lee J-M, Yoon J-H, Cho B-S, Lee S, Kim H-J, Kim M and Kim Y (2021) Integrative Analysis of Gene Expression Data by RNA Sequencing for Differential Diagnosis of Acute Leukemia: Potential Application of Machine Learning.

Front. Oncol. 11:717616 doi: 10.3389/fonc.2021.717616

\section{Integrative Analysis of Gene Expression Data by RNA Sequencing for Differential Diagnosis of Acute Leukemia: Potential Application of Machine Learning}

\author{
Jaewoong Lee ${ }^{1,2}$, Sungmin $\mathrm{Cho}^{3}$, Seong-Eui Hong ${ }^{4}$, Dain $\mathrm{Kang}^{2}$, Hayoung $\mathrm{Choi}^{2}$, \\ Jong-Mi Lee ${ }^{1,2}$, Jae-Ho Yoon ${ }^{5}$, Byung-Sik Cho ${ }^{5}$, Seok Lee ${ }^{5}$, Hee-Je Kim ${ }^{5}$, \\ Myungshin $\mathrm{Kim}^{1,2 * T}$ and Yonggoo Kim ${ }^{1,2 * T}$

\begin{abstract}
${ }^{1}$ Department of Laboratory Medicine, College of Medicine, The Catholic University of Korea, Seoul, South Korea, ${ }^{2}$ Catholic Genetic Laboratory Center, Seoul St. Mary's Hospital, College of Medicine, The Catholic University of Korea, Seoul, South Korea, ${ }^{3}$ Delvine Inc., Seoul, South Korea, ${ }^{4}$ Next Generation Sequencing (NGS) Division, Theragen Bio Co. Ltd., Seongnam-si, South Korea, ${ }^{5}$ Department of Hematology, Catholic Hematology Hospital and Leukemia Research Institute, Seoul St. Mary's Hospital, College of Medicine, The Catholic University of Korea, Seoul, South Korea
\end{abstract}

BCR-ABL1-positive acute leukemia can be classified into three disease categories: B-lymphoblastic leukemia (B-ALL), acute myeloid leukemia (AML), and mixedphenotype acute leukemia (MPAL). We conducted an integrative analysis of RNA sequencing (RNA-seq) data obtained from 12 BCR-ABL1-positive B-ALL, AML, and MPAL samples to evaluate its diagnostic utility. RNA-seq facilitated the identification of all p190 BCR-ABL1 with accurate splicing sites and a new gene fusion involving MAP2K2. Most of the clinically significant mutations were also identified including single-nucleotide variations, insertions, and deletions. In addition, RNA-seq yielded differential gene expression profile according to the disease category. Therefore, we selected 368 genes differentially expressed between $A M L$ and B-ALL and developed two differential diagnosis models based on the gene expression data using 1) scoring algorithm and 2) machine learning. Both models showed an excellent diagnostic accuracy not only for our 12 BCR-ABL1-positive cases but also for 427 public gene expression datasets from acute leukemias regardless of specific genetic aberration. This is the first trial to develop models of differential diagnosis using RNA-seq, especially to evaluate the potential role of machine learning in identifying the disease category of acute leukemia. The integrative analysis of gene expression data by RNA-seq facilitates the accurate differential diagnosis of acute leukemia with successful detection of significant gene fusion and/or mutations, which warrants further investigation.

Keywords: BCR-ABL1, mixed-phenotype acute leukemia, RNA sequencing, gene fusion, mutation, expression, machine learning, acute leukemia 


\section{INTRODUCTION}

Next-generation sequencing (NGS) has been continuously expanded for use in clinical laboratories. It is now commonly used to detect gene mutations in DNA samples and identify recurrent fusions of RNA samples from cancer tissues using applicable cancer panels. Massive parallel sequencing methods using NGS panels are established clinical laboratory tests, which facilitate the detection of significant genetic changes. NGS panels usually include hundreds of genes, but they cannot identify genetic aberrations in unexpected genes. Therefore, recent studies have investigated the application of more extensive NGS platforms such as sequencing of whole genome, whole exome, and transcriptome for clinical cancer genomic profiling (1). Based on such extensive NGS platforms, novel disease categories were defined and recommended. The most representative example is $B C R-A B L 1$-like acute lymphoblastic leukemia (ALL), which was first identified via hierarchical clustering of gene expression profile and a majority of them include gene fusions involving CRLF2, JAK2, and ABL gene categories (2). RNA sequencing (RNA-seq) was routinely used to classify $B C R-A B L 1$-like ALL because it provided transcriptome data including gene expression profiling as well as gene fusions (3). Currently, RNA-seq is extensively used to analyze overall genomic data. Recent studies have improved the utility of RNAseq in identifying gene mutations underlying various cancers, including hematologic malignancies $(4,5)$.

In this study, we performed RNA-seq of acute leukemia samples to evaluate its diagnostic utility. We intentionally selected BCR-ABL1-positive cases, which are recurrent gene fusions found in three categories of acute leukemia: Blymphoblastic leukemia (B-ALL), acute myeloid leukemia (AML), and mixed-phenotype acute leukemia (MPAL). Although all such cases carry the disease-causing BCR-ABL1 fusion, they differ in morphology and antigen expression. Each antigen represents specific hematopoietic lineages, which are usually analyzed by flow cytometry. First, we evaluated the analytical ability of RNA-seq to detect gene fusion and significant mutations. We then analyzed the gene expression data in order to select genes that are differentially expressed between disease categories and identify disease-specific pathways. In addition, we expanded the usage of gene expression data to identify the different disease categories based on the premise that mRNA expression reflects not only disease-specific pathways but also the hematopoietic lineageassociated antigen expression. Toward this end, we developed two models of differential diagnosis based on scoring algorithms and machine learning and verified using public datasets.

\section{METHODS}

\section{Patients and Samples}

We reviewed medical records of patients who were diagnosed with acute leukemia and treated in Seoul St. Mary's Hospital from February 2010 to March 2016. Standard diagnosis was established according to the WHO Classification of Tumours of Haematopoietic and Lymphoid Tissues based on bone marrow (BM) morphology, immunophenotyping, cytogenetic, and molecular genetic analysis (6). Among the consecutive cohorts, 349 patients were $B C R-A B L 1$-positive including B-ALL $(\mathrm{n}=224$, $64.2 \%)$, AML $(\mathrm{n}=10,2.9 \%)$, and MPAL $(\mathrm{n}=9,2.6 \%)$. We selected 12 samples carrying p190 BCR-ABL1 fusions including B-ALL $(n=5)$, AML $(n=3)$, and MPAL $(n=4)$ for further experimental investigation. Their clinical and laboratory characteristics are summarized in Table $\mathbf{1}$.

\section{RNA-Seq and Identification of Fusion and Mutation}

RNAs were extracted from BM samples using RNeasy ${ }^{\circledR}$ Mini Kit (Qiagen, Hilden, Germany). RNA quality was assessed via analysis of rRNA band integrity on an Agilent RNA 6000 Nano Kit (Agilent Technologies, Santa Clara, CA, USA). Prior to cDNA library construction, $1 \mu \mathrm{g}$ of total RNA and magnetic beads with Oligo $(\mathrm{dT})$ were used to enrich poly $(\mathrm{A})$ mRNA. The purified mRNAs were disrupted into short fragments, and the double-stranded cDNAs were immediately synthesized. The cDNAs were subjected to end-repair poly(A) addition and connected with sequencing adapters using the TruSeq RNA Sample Prep Kit (Illumina, San Diego, CA, USA). The fragments automatically purified by BluePippin $2 \%$ agarose gel cassette (Sage Science, Beverly, MA, USA) were selected as templates for PCR amplification. The final library sizes and qualities were evaluated electrophoretically using an Agilent High Sensitivity DNA Kit (Agilent Technologies, Santa Clara, CA, USA) and the fragment size ranged between 350 and $450 \mathrm{bp}$. Subsequently, the library was sequenced using an Illumina HiSeq2500 sequencer (Illumina, San Diego, CA, USA) to obtain 100-bp paired-end reads.

For read mapping and processing, low-quality reads were filtered according to the following criteria: reads containing more than $10 \%$ of skipped bases (marked as 'N's), reads containing more than $40 \%$ of bases whose quality scores were less than 20 , and reads with average quality scores less than 20 each. The whole filtering process was performed using in-house scripts. Filtered reads were then mapped to the human reference genome [Ensembl release 72 (7)] using TopHat, which is supported by Bowtie2 (8). To identify the fusion genes, three different tools [FusionMap (9), nFuse (10), and Chimerascan (11)] were applied to RNA-seq datasets. Finally, the common fusion genes detected by the three tools were considered as actual fusion genes in each patient. To identify variants, GenomeAnalysisTK (v.2.3.9) and Mills-and-1000G-gold.standard-INDELs.hg19 (12) were used for realignment. BaseRecalibrator Tool in Genome Analysis ToolKit (GATK) was used for base quality score recalibration, and variants were called using SNPiR. All variants were then annotated using SnpEff (v.4.1).

\section{Massive Parallel Sequencing for Mutation Detection}

DNAs were extracted from BM samples using QIAamp DNA Mini Kit (Qiagen, Hilden, Germany). DNA quality was assessed 
TABLE 1 | Characteristics of patients at diagnosis.

\begin{tabular}{|c|c|c|c|c|c|}
\hline Case & $\begin{array}{l}\text { Sex/ } \\
\text { Age }\end{array}$ & $\begin{array}{l}\text { WBC } \\
\left(10^{9} / \mathrm{L}\right)\end{array}$ & $\begin{array}{l}\text { BM blast } \\
(\%)\end{array}$ & Positive immunophenotype & Karyotype \\
\hline ALL1 & $\mathrm{F} / 70$ & 13,620 & 92 & CD10, CD19, CD20, CD22, Cy-CD79a, CD34, HLA-DR & $\begin{array}{l}46, X X, t(9 ; 22)(q 34 ; q 11.2)[1] / 45, \text { idem,-7, add(19)(p13.3)[8]/ } \\
46, X X[11]\end{array}$ \\
\hline ALL2 & $\mathrm{F} / 50$ & 3,570 & 99 & CD10, CD19, CD22, Cy-CD79a, CD33, CD34, HLA-DR & $\begin{array}{l}46, X X, t(9 ; 22)(q 34 ; q 11.2)[2] / \\
46, \text { idem,der(20)t(1;20)(q23;q13.1)[10]/46,XX[8] }\end{array}$ \\
\hline ALL3 & $\mathrm{F} / 35$ & 172,530 & 97 & $\begin{array}{l}\text { CD10, CD19, CD20, CD22, Cy-CD79a, CD33, CD34, } \\
\text { HLA-DR }\end{array}$ & $46, X X, t(3 ; 22 ; 9)(p 25 ; q 11.2 ; q 34)[20]$ \\
\hline ALL4 & $\mathrm{M} / 52$ & 50,280 & 80 & CD10, CD19, CD20, CD22, Cy-CD79a, CD34, HLA-DR & $\begin{array}{l}\text { 46,XY,der(9)del(9)(p13p22)t(9;22)(q34;q11.2), der(22)t(9;22) } \\
{[12] / 46, \text { idem, }} \\
\text { del(11)(q11)[7]/46,XY[1] }\end{array}$ \\
\hline ALL5 & $\mathrm{F} / 52$ & 9,260 & 90 & CD10, CD19, CD33, Cy-CD79a, CD34, HLA-DR & 45,XX,der(3;7)(q10;q10),t(9;22)(q34;q11.2)[15]/46,XX[5] \\
\hline AML1 & $\mathrm{F} / 57$ & 89,170 & 75 & $\begin{array}{l}\text { CD33, CD11c, CD14, CD64, CD117, } \\
\text { Cy-MPO, CD10, CD19, CD56, CD34, HLA-DR }\end{array}$ & $46, X X, t(9 ; 22)(q 34 ; q 11.2)[25] / 46, X X[5]$ \\
\hline AML2 & $M / 29$ & 55,530 & 73 & CD33, CD11c, CD117, cy-MPO, CD19, CD34, HLA-DR & $46, X Y, t(9 ; 22)(q 34 ; q 11.2)[20]$ \\
\hline AML3 & $\mathrm{F} / 57$ & 78,670 & 63 & $\begin{array}{l}\text { CD13, CD33, CD11c, CD14, CD64, } \\
\text { Cy-MPO, CD10, CD19, CD22, CD34, HLA-DR }\end{array}$ & $47, X X,+\operatorname{der}(3 ; 17)(q 10 ; q 10), t(9 ; 22 ; 14)(q 34 ; q 11.2 ; q 32)[20]$ \\
\hline MPAL1 & $M / 33$ & 23,290 & 99 & $\begin{array}{l}\text { CD10, CD19, CD79a, Cy-CD22, CD13, CD33, } \\
\text { cy-MPO, CD7, CD34, HLA-DR }\end{array}$ & $45, X Y,-7, t(9 ; 22)(q 34 ; q 11.2)$ \\
\hline MPAL2 & $M / 58$ & 16,510 & 58 & $\begin{array}{l}\text { CD10, CD19, CD79a, Cy-CD22, CD13, CD33, CD11c, } \\
\text { CD64, } \\
\text { Cy-MPO, CD7, CD34, HLA-DR }\end{array}$ & $46, X Y, t(9 ; 22)(q 34 ; q 11.2)$ \\
\hline MPAL3 & $\mathrm{F} / 56$ & 6,820 & 83 & $\begin{array}{l}\text { CD19, CD20, CD79a, Cy-CD22, CD13, CD33, Cy-MPO, } \\
\text { CD34, HLA-DR }\end{array}$ & $\begin{array}{l}\text { 49,XX,+X,+4,+8,t(9;22)(q34;q11.2),i(17)(q10)[5]/49,sl,add(4) } \\
\text { (p16), } \\
\text {-10,add(12)(p11.2),+mar[10]/49,sdl1, del(13)(q14)[2]/48,sdl2, } \\
\text {-del(13q)[2]/50,sl,+8[2]/50,sl,der(3)t(3;?13)(q27; q13),+8[2]/ } \\
\text { 46,XX[4] }\end{array}$ \\
\hline MPAL4 & $\mathrm{F} / 52$ & 43,390 & 87 & $\begin{array}{l}\text { CD10, CD19, CD22, Cy-CD79a, CD33, су- MPO, } \\
\text { CD7, CD34, HLA-DR }\end{array}$ & $45, X X,-7, t(9 ; 22)(q 34 ; q 11.2)[18] / 46, X X[2]$ \\
\hline
\end{tabular}

WBC, while blood cell count; BM, bone marrow; ALL, acute lymphoblastic leukemia; MPAL, mixed-phenotype acute leukemia; AML, acute myeloid leukemia; cy-, cytoplasmic.

with Qubit dsDNA HS Assay Kit (Thermo Fisher Scientific, Waltham, MA, USA). SM acute leukemia panel customized for Seoul St. Mary's Hospital was used to validate the detected mutations via RNA-seq. The SM panel consists of 67 genes with 1,239 DNA amplicons (13). The library preparation was performed via automated processes using IonChef ${ }^{\mathrm{TM}}$ system according to the manufacturer's instructions (Thermo Fisher Scientific, Waltham, MA, USA). Sequencing was performed on an Ion S5 Sequencer (Thermo Fisher Scientific, Waltham, MA, USA). Read mapping, variant calling, and variant annotation were performed using the Ion Torrent Server software. Sequenced reads were mapped to the human reference genome (hg19, Genome Reference Consortium, February 2009) using TMAP v5.2.2. The pathogenic impact on gene function of missense mutations was estimated using in silico prediction of Sorting Intolerant From Tolerant (SIFT) and Polyphen-2. Score $<0.05$ is predicted to be deleterious in SIFT, and score close to 1.0 is predicted to be damaging in PolyPhen-2.

\section{Functional Annotation by Gene Set Enrichment Analysis (GSEA) and Gene Ontology (GO)}

The gene expression level was measured with Cufflinks v2.1.1 (14) using the gene annotation database of Ensembl release 72. The non-coding region was removed. Multiread correction and frag-bias-correct were used to improve the accuracy of measurement. Differentially expressed genes (DEGs) were identified using Cuffdiff tool with a statistically significant $q$-value $(<0.05)$. First, we performed functional annotation of DEGs according to the GO Consortium (http://www. geneontology.org/index.shtml) by R package, goseq (15). GO database classifies genes according to the three categories of biological process (BP), cellular component (CC), and molecular function (MF) and annotates the function of the selected genes. $P$ values $<0.001$ were considered statistically significant. This ontology result was rechecked using the database for annotation, visualization, and integrated discovery (DAVID) bioinformatics tool (16). In addition, we performed GSEA to analyze the critical transcriptome pathways (17). Toward this end, the estimated expression levels were used in GSEA to determine the enrichment scores according to the rankedordered gene list. With the predefined gene sets of GSEA, a total of 167 Kyoto Encyclopedia of Genes and Genomes (KEGG) pathways were considered, and the pathways containing at least 15 genes were evaluated. The significant scores were computed using 1,000 nonparametric permutation test, and $P$ values $<0.05$ were considered as statistically significant.

\section{Integration of Gene Network Analysis}

We uploaded the DEG lists containing gene identifiers (probe set IDs) and corresponding $P$-values to Ingenuity Pathway Analysis (IPA; Ingenuity Systems, www.ingenuity.com). The association of the gene sets with the canonical pathways indicated possible effects on the well-defined biological pathway of the IPA platform based on the most up-to-date knowledge base. We extracted the most drastically affected functions $(P$-value $\leq 0.05$; 
$\mid z$-score $\mid \geq 2$ ). The right-tailed Fisher's exact test was used to estimate the probability that the association between a set of molecules and a function or pathway might be due to random chance. We also generated protein-protein interaction networks and a network view, which revealed the molecular relationship between the molecules (18).

\section{Development of Differential Diagnosis Models; Scoring Algorithm and Machine Learning}

The differential diagnosis of acute leukemia is based on antigen expression on the surface and in the cytoplasm, which are usually analyzed by flow cytometry. Because the antigen expression was closely associated with gene expression, we postulated that the three categories of acute leukemia can be distinguished via analysis of gene expression data. Therefore, we selected DEGs, which showed more than 2-fold expression and a significant $q$-value $(<0.05)$ in AML compared with B-ALL, and defined them as AML-specific genes. B-ALL-specific genes were defined similarly. We limited genes with at least 100 fragments per kilobase of transcript per million mapped reads (FPKM). Utilizing these tools, we finally selected 251 AML- and 117 BALL-specific genes (Supplementary Table S1). Each gene showed a different FPKM value. Thus, it is necessary to set a threshold value to define whether or not the gene was positively expressed. We carefully set the threshold and developed an analytical approach for application of the model to any gene expression data such as microarray as well as RNA-seq of acute leukemia cases. The details of the process and the formula are described in the Supplementary Materials. When the FPKM value of one gene was greater than the final threshold, it was included in the number of positively expressed genes. We obtained the AML and B-ALL scores based on the proportion of positively expressed AML- and B-ALL-specific genes, respectively. As expected, the AML score was the highest in AML followed by MPAL- and B-ALL-specific genes, and the BALL score was in the reverse order (Supplementary Figure S1A). We classified AML, B-ALL, and MPAL based on two scores according to the following criteria: 1) AML when AML score $\geq 30$ and B-ALL score < 30, 2) B-ALL when AML score < 30 and B-ALL score $\geq 30$, and 3) MPAL when both AML and BALL scores $\geq 30$. Repeated simulations were performed to optimize the differential diagnosis scoring algorithm to obtain a diagnostic accuracy of $92 \%$ (11/12, Supplementary Figure S1B).

In addition, we conducted machine learning for differential diagnosis using gene expression data selected for the scoring algorithm. The soft margin support vector machine (SVM) (19) was used because the gene expression data were not linearly separable. Kernel function of hyperbolic tangent was applied for SVM. The Z-transformation of each disease category was carried out for standardization. We selected the one-vs.-one strategy, which splits multiple classes into single binary elements for each pair of classes (e.g., B-ALL-vs.-AML). Although the sample size was small to generate enough datasets for machine learning, we performed one-vs.-all SVM cross-validation to reduce this limitation. This method repeated the analyses using data from 1 case for validation and the other 11 for learning, which is described in the Supplementary Materials. The cross-validation of our SVM model of machine learning based on our 12 cases revealed 100\% diagnostic accuracy, sensitivity, and specificity.

\section{Validation of Differential Diagnosis Models Using Public Datasets}

To validate the models for differential diagnosis of acute leukemia, we collected 427 public gene expression datasets from the International Cancer Genome Consortium (ICGC) Data Portal (dcc.icgc.org; 206 samples of ALL-US project EXPA data and 197 samples of LAML-US project EXP-A data) and the National Center for Biotechnology Information (NCBI) website (www.ncbi.nlm.nih.gov; 24 samples of Series GSE113601 dataset). Reference gene ( $A B L 1)$ was used as the internal reaction control to normalize the gene expression. AML and B-ALL scores were calculated as described previously. We also validated the performance of a machine learning method using normalized gene expression levels of the same public dataset.

\section{Statistical Analysis}

Statistical differences between groups were determined using oneway analysis of variance (ANOVA) followed by Bonferroni's post hoc test for multiple comparisons. Pearson's correlation analysis was performed for the quantitative results of variant allele frequencies (VAFs) from RNA-seq with those from massive parallel sequencing. All analyses were performed using IBM $^{\circledR}$ SPSS $^{\circledR}$, version 24.0 (IBM Corp., Armonk, NY, USA). Differences were significant at $P<0.05$.

\section{RESULTS}

\section{Detection of the Gene Fusions and Mutations by RNA-Seq}

RNA-seq revealed $B C R-A B L 1$ gene fusions in all enrolled cases. The number of fusion transcripts varied in each case: 1 to 4 for $B C R-A B L 1$ and 0 to 3 for $A B L 1-B C R$. All cases carried a BCR$A B L 1$ fusion transcript with a splicing site of chr22:23524426 and chr9:133729451. The secondary splicing site located on the three base pairs differed from the first $A B L 1$ splicing site as chr9:133729454, which was observed in five cases. $A B L 1-B C R$ fusion transcripts were detected in 10 cases with a shared splicing site in chr9:133589842 (exon 1a) and chr22:23595986. The second common splicing site was located on chr9:133710912 (exon 1b) with the same BCR splicing site. No differences in splicing sites were detected in cases according to the disease category (Supplementary Figure S2).

In addition, we found a new fusion in a B-ALL patient with $\operatorname{der}(19) \mathrm{t}(7 ; 19)(\mathrm{p} 14 ; \mathrm{p} 13.3)$; mitogen-activated protein kinase kinase 2 (MAP2K2)-AC010132.5. The MAP2K2 gene, located on 19p13.3, is a dual-specificity protein kinase belonging to the MAP kinase kinase family. This kinase is known to play a critical role in mitogen growth factor signal transduction. AC010132.5 is 
located on $7 \mathrm{p} 14$. This fusion was confirmed by Reverse Transcription Polymerase Chain Reaction (RT-PCR) and Sanger sequencing (Supplementary Figure S3).

DNA-based SM NGS panel detected 11 single-nucleotide variations, two insertions, and one in-frame deletion. RNA-seq detected these variants except two on the NOTCH3 gene because of the low gene expression value as FPKM $<1.0$ (Table 2). VAFs from RNA-seq showed good correlation with those from massive parallel sequencing except mutations with low FPKM $<5.0(\mathrm{r}=$ $0.787, P=0.004)$. We also analyzed the point mutations within the $A B L 1$ kinase domain associated with drug resistance and found no significant mutation.

\section{Different Gene Expression Profiles Among AML, B-ALL, and MPAL}

We identified significantly different GO profiles among the three disease categories including mitogen-activated protein kinase (MAPK) cascade, angiogenesis, cell activation, cytokine production, regulation of protein phosphorylation, immune system process, leukocyte differentiation, receptor activity, receptor binding, cell communication and adhesion, and cell death and proliferation (Supplementary Table S2). When comparing AML with B-ALL, the genes associated with extracellular exosome, extracellular space, innate immune response, defense response to bacterium, Fc-gamma receptor signaling pathway involved in phagocytosis, plasma membrane, phagocytosis, and engulfment serine-type endopeptidase activity were enriched in AML. The genes associated with cytoplasm, B cell differentiation, and cell proliferation were enriched in BALL. Compared with the other leukemias, MPAL revealed higher gene expression of RNA- and translation-related ontologies (Figure 1).

In terms of GSEA, the KEGG pathway analyses of 167 gene sets showing significantly different expression between AML and B-ALL identified 118 upregulated gene sets in AML and 49 upregulated in B-ALL. Among the upregulated gene sets in AML, 29 gene sets showed false discovery rate (FDR) $<0.25$ and a nominal $P$ value $<0.01$ (Supplementary Table S3). Among the upregulated gene sets in B-ALL, two gene sets showed significant FDR and nominal $P$ value. The IPA analysis of these gene sets indicated the major altered canonical pathways in MPAL including IL-6 signaling, PPAR signaling, 14-3-3-mediated signaling, osteoarthritis pathway, D-myo-inositol-5-phosphate metabolism, antioxidant action of vitamin $\mathrm{C}$, regulation of the epithelial mesenchymal transition by growth factors pathway, super pathway of inositol phosphate compounds, cardiac hypertrophy signaling (enhanced), and PI3K signaling in B lymphocytes (Supplementary Figure S4).

\section{Predictive Performance of Developed Differential Diagnosis Models}

We analyzed the public gene expression datasets and compared the AML and B-ALL scores of three disease categories. The AML score was significantly high in AML and MPAL compared with B-ALL $(P<0.001$ and $<0.001$, respectively). The B-ALL score was significantly high in B-ALL followed by MPAL and AML ( $P<0.001$ for each comparison) (Figure 2A). In the scattergram of B-ALL score and AML score, the MPAL samples cluster between AML and B-ALL samples (Figure 2B). The diagnostic accuracy of scoring algorithm and machine learning was $97.2 \%$ (415/427) and 99.1\% (423/427), respectively. We analyzed additional predictive performance of two differential diagnosis models according to disease category. The diagnostic sensitivities of the scoring algorithm were $99.0 \%, 95.4 \%$, and $95.8 \%$ in B-ALL, AML, and MPAL, respectively. Those of the machine learning were $99.5 \%, 99.5 \%$, and $91.7 \%$ in the same order, respectively. The specificities of the scoring algorithm were $95.5 \%, 98.7 \%$, and 97.3\% in B-ALL, AML, and MPAL, respectively. And those of the machine learning were $100 \%, 98.7 \%$, and $99.8 \%$ in the same order, respectively (Supplementary Table S4).

TABLE 2 | Mutations analyzed by massive parallel sequencing of DNA and RNA sequencing.

\begin{tabular}{|c|c|c|c|c|c|c|c|c|c|c|c|}
\hline Case & Gene & Transcript & Base change & AA Change & Type of mutation & VAF $^{*}$ & RNA-seq & VAF $^{\star *}$ & FPKM $^{\star \star *}$ & SIFT $^{\dagger}$ & Polyphen-2 $^{\dagger}$ \\
\hline ALL1 & SETD2 & NM_014159.6 & c.1409_1410insGCCC & $\mathrm{R} 471 \mathrm{Pfs}^{\star} 21$ & frameshift & 29.45 & Detected & 33.33 & 40.51 & - & - \\
\hline ALL3 & PAX5 & NM_016734.2 & c.55_56insTG & G19Vfs*3 & frameshift & 38.34 & Detected & 29.81 & 238.84 & - & - \\
\hline ALL4 & NOTCH3 & NM_000435.2 & c.3736G >A & V1246I & missense & 50.33 & Not detected & - & 0.4 & 0.52 & 0 \\
\hline ALL4 & TET2 & NM_001127208.2 & C.3116C>T & S1039L & missense & 53.55 & Detected & 34.48 & 10.6 & 0.29 & 0.968 \\
\hline ALL4 & PAX5 & NM_016734.2 & c.70G >C & G24R & missense & 6.37 & Detected & 13.64 & 78.66 & 0 & 1.000 \\
\hline AML1 & NOTCH3 & NM_000435.2 & c. $709 \mathrm{G}>\mathrm{A}$ & V237M & missense & 52.36 & Detected & - & 1.04 & 0 & 0.908 \\
\hline AML2 & NOTCH3 & NM_000435.2 & c.33_35delCCG & R12del & in-frame deletion & 4.62 & Not detected & - & 0.79 & - & - \\
\hline AML2 & RUNX1 & NM_001754.4 & c. $610 \mathrm{C}>\mathrm{T}$ & R204* & nonsense & 43.35 & Detected & 52.94 & 105.24 & - & - \\
\hline AML2 & $R \cup N X 1$ & NM_001754.4 & c. $601 \mathrm{C}>\mathrm{T}$ & $\mathrm{R} 201^{*}$ & nonsense & 45.35 & Detected & 44.20 & 105.24 & - & - \\
\hline AML3 & NF1 & NM_001042492.2 & c. $4379 A>G$ & H1460R & missense & 65.3 & Detected & 68.57 & 27.73 & 0.27 & 0.048 \\
\hline MPAL2 & TET2 & NM_001127208.2 & c. $2604 \mathrm{~T}>\mathrm{G}$ & F868L & missense & 53.33 & Detected & 63.64 & 14.54 & 0.22 & 0.307 \\
\hline MPAL2 & NF1 & NM_001042492.2 & c. $1198 \mathrm{C}>\mathrm{G}$ & Q400E & missense & 45.9 & Detected & 61.54 & 6.1 & 1 & 0.008 \\
\hline MPAL4 & SH2B3 & NM_005475.2 & C. $1139 T>C$ & L380P & missense & 47.95 & Detected & 42.86 & 39.96 & 0.2 & 0.966 \\
\hline MPAL4 & NOTCH3 & NM_000435.2 & c. $709 \mathrm{G}>\mathrm{A}$ & V237M & missense & 48.29 & Detected & 66.67 & 1.09 & 0 & 0.908 \\
\hline
\end{tabular}

RNA-seq detected most variants detected in DNA sequencing except two with low gene expression value as FPKM $<1.0$.

AA, amino acid; VAF, percentage of variant allele frequency; RNA-seq, RNA sequencing; FPKM, Fragment Per Kilobase of transcript per Million mapped reads; SIFT, Sorting Intolerant From Tolerant.

*VAF obtained from massive parallel sequencing of DNA.

${ }^{*} V A F$ and FPKM obtained from RNA-seq.

${ }^{t}$ The SIFT and Polyphen2 in-silico scores are unavailable for frameshift and nonsense mutations and in-frame deletion. 


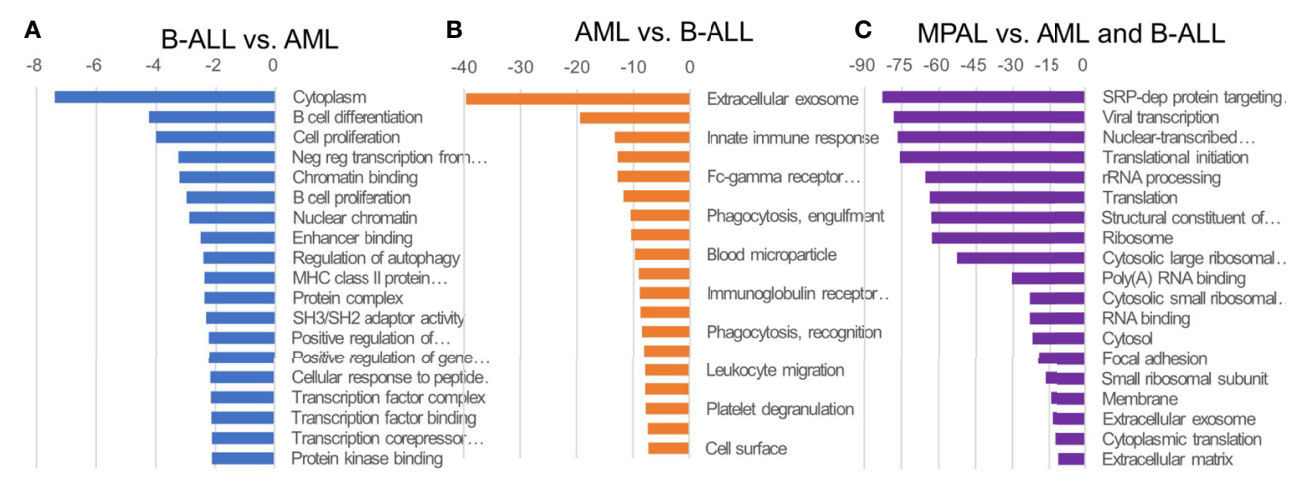

FIGURE 1 | GO of ALL, AML, and MPAL. General functional classification of highly expressed gene in B-lymphoblastic leukemia (B-ALL) compared to acute myeloid leukemia (AML) (A), AML compared to B-ALL (B), and in mixed-phenotype acute leukemia (MPAL) compared to B-ALL and AML (C). Gene Ontology (GO) analysis within target genes of significantly altered transcripts was performed using the database for annotation, visualization and integrated discovery (DAVID) bioinformatics tool. Enriched GO biological processes were identified and listed according to their enrichment $P$ value $(P<0.05)$ and false discovery rate (FDR $<0.25)$. Both $P$ and FDR values were obtained using DAVID 2.1 statistical function classification tool. scale: - $\log _{10}$ of $p$-value.

\section{DISCUSSION}

In the current study, we performed RNA-seq of 12 acute leukemia cases carrying p190 BCR-ABL1. The analysis of three disease categories using various angles facilitated the comprehensive evaluation of RNA-seq performance in gene fusion, mutation, and gene expression and the application of expression data to differential diagnosis. RNA-seq not only enabled the reliable detection of all $B C R-A B L 1$ but also provided accurate splicing sites. There was a common splicing site of $B C R$ and $A B L 1$ genes as chr22:23524426 and chr9:133729451. Some patients carried more than one $B C R-A B L 1$ fusions. $A B L 1-B C R$ fusion was generated in some patients. Notably, a new fusion was detected in this study. Gene fusions in acute leukemia are implicated in the onset of malignancies, and recurrent driver gene fusions are used in the classification. Novel gene fusions have been continuously discovered in hematologic malignancies and represent potential diagnostic or therapeutic targets (20, 21). RNA-seq is a useful tool for genome-wide surveillance of gene fusions with nucleotide-level resolution of fusion junctions (22). Studies have demonstrated the potential to identify gene fusions via improved bioinformatics workflows (23-25), and therefore, RNA-seq can be used not only to identify driver gene fusions but also to detect novel and rare gene fusions in clinical laboratories.

Gene mutations are usually analyzed via DNA sequencing. However, we postulated that RNA-seq facilitates the screening of clinically significant mutations based on sequencing data. The current and previous studies have shown that somatic mutations can be identified in cancer based on RNA-seq data of cancerrelated genes $(4,26)$. A specific software, i.e., RNAmut, which enabled the detection of all clinically important mutations
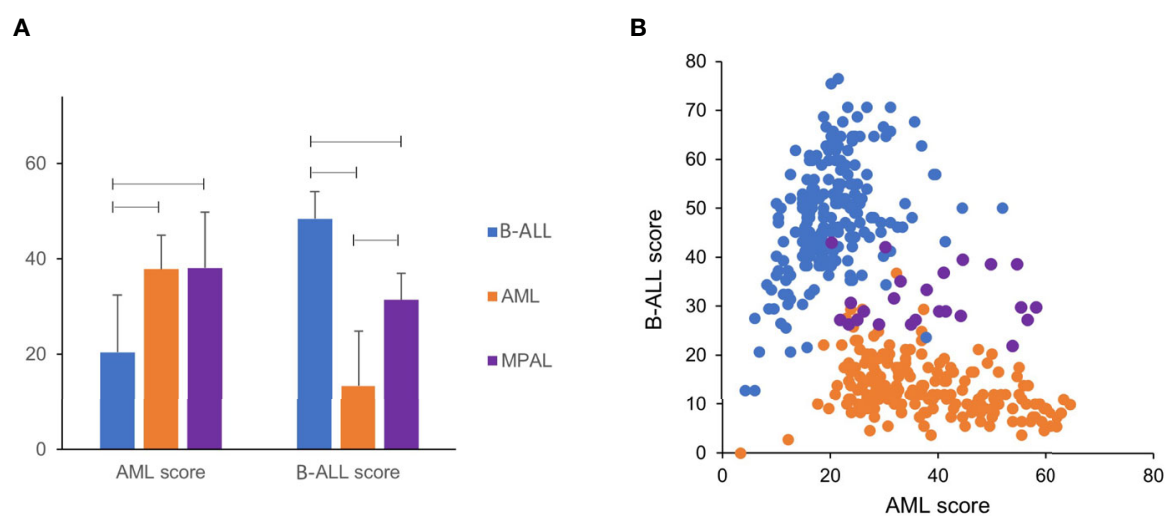

FIGURE 2 | AML score and B-ALL score of public data. (A) Except AML score between AML and MPAL, AML score and B-ALL score between each leukemia were significantly different $(p<0.01)$. (B) Public data of $A M L$ and B-ALL show clustering in scatter plot of AML score and B-ALL score. MPAL samples cluster between $\mathrm{AML}$ and B-ALL samples. 
in AML, was developed (5). And tandem duplications in FLT3 and KMT2A were also effectively detected by RNA-seq using those novel algorithms $(5,27)$. Integrated genomic profiling has increased the ability to identify clinically relevant genomic alterations of therapeutic significance in hematologic malignancies (28). In the current study, we successfully detected clinically significant somatic mutations using RNA-seq data except the mutations in the genes with very low transcript. Also, their VAFs from RNA-seq showed good correlation with those from massive parallel sequencing. Additionally, we identified mutations within the $A B L 1$ kinase domain via RNAseq, which was associated with drug resistance to tyrosine kinase inhibitors. However, the study showed an intrinsic limitation in that poorly expressed gene mutations could not be analyzed by RNA-seq.

Gene expression data showed significantly different GO profiles among the three disease categories. In B-ALL, B cell differentiation and transcription factor-associated GOs were enriched. The result was in agreement with previous studies that demonstrated that malignant conversion of B-lymphocyte progenitors involves multiple targeting of a central transcription factor network (29). In AML, GOs associated with functions of granulocytes were enriched such as phagocytosis, lysosome, and innate immune response. Also, results from this and previous studies showed that extracellular exosome was enriched in AML, which express the properties relevant to AML pathogenesis that affected prognosis, response to therapy, and leukemic niche formation (30). The MAPK cascade is one of the differential GO profiles that plays an essential role in connecting cell-surface receptors with altered transcriptional programs and aberrant MAPK activation in the pathogenesis of various myeloid malignancies (31). In addition, the stress-activated MAPK pathways influence the response of cancer cells to chemotherapies and targeted therapies (32). Therefore, the role of different ontology profiles in each disease category requires further elucidation.

We investigated MPAL-specific pathways via substantial analysis of gene expression profiles and found out that MPAL constituted a heterogeneous group of diseases rather than induced by a specific pathway. MPAL is acute leukemia of ambiguous lineage that is defined by antigen expression. MPAL contains blasts that express antigens of more than one lineage to such a degree that is not possible to attribute the leukemia to any one lineage definitively. Other evidence suggests that the cell of origin in B-ALL associated with $B C R-A B L 1$ was more immature than that of other B-ALL cases (33). A hematopoietic progenitor cell with multilineage potential is considered as a normal counterpart of the leukemic cells in AML and MPAL with $B C R-A B L 1$ (33). Therefore, we carefully postulated that acute leukemia with $B C R-A B L 1$ may represent a series of diseases carrying different proportions of specific lineage.

This is the first trial to develop models of differential diagnosis using RNA-seq. Based on AML and ALL-specific gene expression data, we developed two models, namely, scoring algorithm and machine learning. Although there was a practical limitation of small sample size for machine learning, both models were effective not only in our 12 BCR-ABL1- positive cases but also in public datasets from acute leukemias regardless of specific genetic aberration. In addition, gene expression data contain profound information including molecular pathways associated with disease pathogenesis and potential therapeutic targets. Recently, the usage of gene expression data has been expanded by integrating traditional medical data and advanced rapidly using machine learning algorithms in real clinical medicine (13). The machine learning model discussed in this study might be used to integrate gene expression data with gene fusions and mutations.

Taken together, this study demonstrated that RNA-seq not only enabled the detection of gene fusion and clinically significant mutations but also assigned the lineage of acute leukemia according to gene expression. The potential application of gene expression data by RNA-seq data that facilitate the accurate differential diagnosis of acute leukemia requires further investigation.

\section{DATA AVAILABILITY STATEMENT}

RNA-seq data generated in this study is available at NCBI BioProject database: https://www.ncbi.nlm.nih.gov/bioproject/PRJNA733693. Gene expression data of ACUTE LYMPHOBLASTIC LEUKEMIA TARGET, US is available at ICGC Data Portal: https://dcc.icgc.org/ projects/ALL-US. Gene expression data of ACUTE MYELOID LEUKEMIA - TCGA, US is available at ICGC Dapa Portal: https:// dcc.icgc.org/projects/LAML-US. Gene expression data of RNA sequencing analysis of adult mixed phenotype acute leukemia (MPAL) is available at NCBI GEO: https://www.ncbi.nlm.nih.gov/ geo/query/acc.cgi?acc=GSE113601.

\section{ETHICS STATEMENT}

The study protocol was approved by the Institutional Review Board of The Catholic University of Korea (No.KC16SISI1026) and performed in accordance with the Declaration of Helsinki.

\section{AUTHOR CONTRIBUTIONS}

MK and YK were responsible for the study concept and project administration. J-HY, B-SC, SL and H-JK acquired resources. JL, SC, S-EH, J-ML, DK and HC analyzed and interpreted data. JL drafted the manuscript. All authors contributed to the article and approved the submitted version.

\section{FUNDING}

This research was funded by Ministry of Food and Drug Safety (grant number 18172MFDS182) and the National Research Foundation of Korea (NRF) funded by the Korea government (MSIT) (grant number 2020R1F1A1068437). 


\section{ACKNOWLEDGMENTS}

This research was supported by Catholic Genetics Laboratory Center, Seoul St. Mary's Hospital, the Catholic University of Korea.

\section{REFERENCES}

1. Rusch M, Nakitandwe J, Shurtleff S, Newman S, Zhang Z, Edmonson MN, et al. Clinical Cancer Genomic Profiling by Three-Platform Sequencing of Whole Genome, Whole Exome and Transcriptome. Nat Commun (2018) 9:3962. doi: 10.1038/s41467-018-06485-7

2. Roberts KG, Li Y, Payne-Turner D, Harvey RC, Yang YL, Pei D, et al. Targetable Kinase-Activating Lesions in Ph-Like Acute Lympho blastic Leukemia. N Engl J Med (2014) 371:1005-15. doi: 10.1056/NEJMoa 1403088

3. Den Boer ML, van Slegtenhorst M, De Menezes RX, Cheok MH, Buijs-Gladdines JG, Peters ST, et al. A Subtype of Childhood Acute Lymphoblastic Leukaemia With Poor Treatment Outcome: A GenomeWide Classification Study. Lancet Oncol (2009) 10:125-34. doi: 10.1016/ S1470-2045(08)70339-5

4. Coudray A, Battenhouse AM, Bucher P, Iyer VR. Detection and Benchmarking of Somatic Mutations in Cancer Genomes Using RNA-Seq Data. PeerJ (2018) 6:e5362. doi: 10.7717/peerj.5362

5. Gu M, Zwiebel M, Ong SH, Boughton N, Nomdedeu J, Basheer F, et al. RNAmut: Robust Identification of Somatic Mutations in Acute Myeloid Leukemia Using RNA-Sequencing. Haematologica (2020) 105:e290-e3. doi: 10.3324/haematol.2019.230821

6. Steven HS, Elias C, Nancy LH, Elaine SJ, Stefano AP, Harald S, et al. WHO Classification of Tumours of Haematopoietic and Lymphoid Tissues Revised 4th Edition. Lyon, France: International Agency for Research on Cancer (IARC) (2017).

7. Flicek P, Ahmed I, Amode MR, Barrell D, Beal K, Brent S, et al. Ensembl 2013. Nucleic Acids Res (2013) 41:D48-55. doi: 10.1093/nar/gks236

8. Langmead B, Salzberg SL. Fast Gapped-Read Alignment With Bowtie 2. Nat Methods (2012) 9:357-9. doi: 10.1038/nmeth.1923

9. Ge H, Liu K, Juan T, Fang F, Newman M, Hoeck W. FusionMap: Detecting Fusion Genes From Next-Generation Sequencing Data at Base-Pair Resolution. Bioinformatics (2011) 27:1922-8. doi: 10.1093/bioinformatics/ btr310

10. Lathi RB, Massie JA, Loring M, Demko ZP, Johnson D, Sigurjonsson S, et al. Informatics Enhanced SNP Microarray Analysis of 30 Miscarriage Samples Compared to Routine Cytogenetics. PLoS One (2012) 7:e31282. doi: 10.1371/ journal.pone.0031282

11. Iyer MK, Chinnaiyan AM, Maher CA. ChimeraScan: A Tool for Identifying Chimeric Transcription in Sequencing Data. Bioinformatics (2011) 27:2903-4. doi: 10.1093/bioinformatics/btr467

12. McKenna A, Hanna M, Banks E, Sivachenko A, Cibulskis K, Kernytsky A, et al. The Genome Analysis Toolkit: A MapReduce Framework for Analyzing Next-Generation DNA Sequencing Data. Genome Res (2010) 20:1297-303. doi: 10.1101 /gr.107524.110

13. Myszczynska MA, Ojamies PN, Lacoste AMB, Neil D, Saffari A, Mead R, et al. Applications of Machine Learning to Diagnosis and Treatment of Neurodegenerative Diseases. Nat Rev Neurol (2020) 16:440-56. doi: 10.1038/s41582-020-0377-8

14. Trapnell C, Roberts A, Goff L, Pertea G, Kim D, Kelley DR, et al. Differential Gene and Transcript Expression Analysis of RNA-Seq Experiments With TopHat and Cufflinks. Nat Protoc (2012) 7:562-78. doi: 10.1038/ nprot.2012.016

15. Young MD, Wakefield MJ, Smyth GK, Oshlack A. Gene Ontology Analysis for RNA-Seq: Accounting for Selection Bias. Genome Biol (2010) 11:R14. doi: 10.1186/gb-2010-11-2-r14

16. Dennis GJr., Sherman BT, Hosack DA, Yang J, Gao W, Lane HC, et al. DAVID: Database for Annotation, Visualization, and Integrated Discovery. Genome Biol (2003) 4:P3. doi: 10.1186/gb-2003-4-9-r60

\section{SUPPLEMENTARY MATERIAL}

The Supplementary Material for this article can be found online at: https://www.frontiersin.org/articles/10.3389/fonc.2021.717616/ full\#supplementary-material

17. Subramanian A, Tamayo P, Mootha VK, Mukherjee S, Ebert BL, Gillette MA, et al. Gene Set Enrichment Analysis: A Knowledge-Based Approach for Interpreting Genome-Wide Expression Profiles. Proc Natl Acad Sci U S A (2005) 102:15545-50. doi: 10.1073/pnas.0506580102

18. Krämer A, Green J, Pollard JJr., Tugendreich S. Causal Analysis Approaches in Ingenuity Pathway Analysis. Bioinformatics (2014) 30:523-30. doi: 10.1093/ bioinformatics/btt703

19. Cortes C, Vapnik V. Support-Vector Networks. Mach Learn (1995) 20:27397. doi: 10.1007/BF00994018

20. Mata-Rocha M, Rangel-López A, Jiménez-Hernández E, Morales-Castillo BA, González-Torres C, Gaytan-Cervantes J, et al. Identification and Characterization of Novel Fusion Genes With Potential Clinical Applications in Mexican Children With Acute Lymphoblastic Leukemia. Int J Mol Sci (2019) 20(10):2394. doi: 10.3390/ijms20102394

21. Padella A, Simonetti G, Paciello G, Giotopoulos G, Baldazzi C, Righi S, et al. Novel and Rare Fusion Transcripts Involving Transcription Factors and Tumor Suppressor Genes in Acute Myeloid Leukemia. Cancers (Basel) (2019) 11(12):1951. doi: 10.3390/cancers11121951

22. Ozsolak F, Milos PM. RNA Sequencing: Advances, Challenges and Opportunities. Nat Rev Genet (2011) 12:87-98. doi: 10.1038/nrg2934

23. Mittal VK, McDonald JF. De Novo Assembly and Characterization of Breast Cancer Transcriptomes Identifies Large Numbers of Novel Fusion-Gene Transcripts of Potential Functional Significance. BMC Med Genomics (2017) 10:53. doi: 10.1186/s12920-017-0289-7

24. Heyer EE, Deveson IW, Wooi D, Selinger CI, Lyons RJ, Hayes VM, et al. Diagnosis of Fusion Genes Using Targeted RNA Sequencing. Nat Commun (2019) 10:1388. doi: 10.1038/s41467-019-09374-9

25. Lee JW, Kim Y, Cho B, Kim S, Jang PS, Lee J, et al. High Incidence of RAS Pathway Mutations Among Sentinel Genetic Lesions of Korean Pediatric BCRABL1-Like Acute Lymphoblastic Leukemia. Cancer Med (2020) 9:4632-9. doi: 10.1002/cam4.3099

26. Wolff A, Perera-Bel J, Schildhaus HU, Homayounfar K, Schatlo B, Bleckmann A, et al. Using RNA-Seq Data for the Detection of a Panel of Clinically Relevant Mutations. Stud Health Technol Inform (2018) 253:217-21. doi: 10.3233/978-1-61499-896-9-217

27. Arindrarto W, Borràs DM, de Groen RAL, van den Berg RR, Locher IJ, van Diessen SAME, et al. Comprehensive Diagnostics of Acute Myeloid Leukemia by Whole Transcriptome RNA Sequencing. Leukemia (2021) 35:47-61. doi: 10.1038/s41375-020-0762-8

28. He J, Abdel-Wahab O, Nahas MK, Wang K, Rampal RK, Intlekofer AM, et al. Integrated Genomic DNA/RNA Profiling of Hematologic Malignancies in the Clinical Setting. Blood (2016) 127:3004-14. doi: 10.1182/blood-2015-08664649

29. Tomar AK, Agarwal R, Kundu B. Most Variable Genes and Transcription Factors in Acute Lymphoblastic Leukemia Patients. Interdiscip Sci (2019) 11:668-78. doi: 10.1007/s12539-019-00325-y

30. Kang K, Kim H, Hur W, Jung J, Jeong JH, Shin H, et al. A Proteomic Approach to Understand the Clinical Significance of Acute Myeloid Leukemia-Derived Extracellular Vesicles Reflecting Essential Characteristics of Leukemia. Mol Cell Proteomics (2020) 20:100017. doi: 10.1074/mcp.RA120.002169

31. Geest CR, Coffer PJ. MAPK Signaling Pathways in the Regulation of Hematopoiesis. J Leukoc Biol (2009) 86:237-50. doi: 10.1189/jlb.0209097

32. Lee S, Rauch J, Kolch W. Targeting MAPK Signaling in Cancer: Mechanisms of Drug Resistance and Sensitivity. Int J Mol Sci (2020) 21(3):1102. doi: 10.3390/ijms21031102

33. Steven HS, Elias C, Nancy LH, Elaine SJ, Stefano AP, Harald S, et al. WHO Classification of Tumours of Haematopoietic and Lymphoid Tissues Revised 4th Edition. Lyon, France: International Agency for Research on Cancer (IARC) (2017). p. 140, 182, 203. 
Conflict of Interest: Author SC is the CEO of Delvine Inc. Author SH is employed by Theragen Bio Co. Ltd.

The remaining authors declare that the research was conducted in the absence of any commercial or financial relationships that could be construed as a potential conflict of interest.

Publisher's Note: All claims expressed in this article are solely those of the authors and do not necessarily represent those of their affiliated organizations, or those of the publisher, the editors and the reviewers. Any product that may be evaluated in this article, or claim that may be made by its manufacturer, is not guaranteed or endorsed by the publisher.

Copyright $\odot 2021$ Lee, Cho, Hong, Kang, Choi, Lee, Yoon, Cho, Lee, Kim, Kim and Kim. This is an open-access article distributed under the terms of the Creative Commons Attribution License (CC BY). The use, distribution or reproduction in other forums is permitted, provided the original author(s) and the copyright owner(s) are credited and that the original publication in this journal is cited, in accordance with accepted academic practice. No use, distribution or reproduction is permitted which does not comply with these terms. 